\title{
TRES HOMBRES DE CIENCIA EN LA CONSTITUCIÓN DEL OBJETO GEOLÓGICO EN MÉXICO
}

\author{
THREE MEN OF SCIENCE IN THE CONSTITUTION OF THE GEOLOGICAL \\ OBJECT IN MEXICO
}

\begin{abstract}
José Alfredo Uribe Salas ${ }^{(*)}$
Universidad Michoacana de San Nicolás de Hidalgo Morelia, Michoacán (MN), México

María Teresa Cortés Zavala ${ }^{(* *)}$

Universidad Michoacana de San Nicolás de Hidalgo Morelia, Michoacán (MN), México
\end{abstract}

Resumen: El artículo analiza la relación intrínseca entre educación y ciencia en el proceso de institucionalización de la mineralogía y la geología en México del siglo XIX. Destacamos el liderazgo intelectual de tres hombres de ciencia: Andrés Manuel del Río, Antonio del Castillo y José G. Aguilera, quienes representan el soporte del proceso de institucionalización y profesionalización de las Ciencias de la Tierra, a través del Real Seminario de Minería, Escuela de Minería y Escuela Nacional de Ingenieros, para terminar en el Instituto de Geológico Nacional, antecedente directo Instituto de Geología de la Universidad Nacional Autónoma de México.

Palabras claves: Geología; Institucionalización; México.

Abstract: This article analyzes the relation between education and science in the process of institutionalization of mineralogy and geology in nineteenth-century Mexico. It focuses on the intellectual leadership of three men: Andrés Manuel del Río, Antonio del Castillo y José G. Aguilera. The work of these men represents the foundation of the process of institutionalization and professionalization of Earth Sciences in Mexico through the Royal Mining Seminar, the Mining School, the National School of Engineering and the National Institute of Geology the immediate antecedent of the modern Institute of Geology at the National Autonomous University of Mexico.

Keywords: Geology; Institucionalization; Mexico.

(*) Doctor, Profesor investigador de la Faculdad de Historia de la Universidad Michoacana de San Nicolás de Hidalgo. E-mail: <jausalas@gmail.com>

(*) Doctora, Profesora investigadora da la Faculdad de Historia Universidad Michoacana de San Nicolás de Hidalgo. E-mail: <mtczavala@gmail.co>. Recebidoem 23.04.2104, aceptado en: 02.10.2014. 


\section{INTRODUCCIÓN}

Desde finales del siglo XVIII y a lo largo del XIX estuvo presente entre los gobiernos el imperativo de modernizar las formas del Estado y los procesos productivos. Tanto en el virreinato de la Nueva España como en el México independiente, los diferentes gobiernos y grupos de poder económico se plantearon la alternativa de modernizar el motor de la economía, representado por la industria minero metalúrgica, sobre la base de integrar y fortalecer la presencia de una comunidad científica activa capas de orientar con sus saberes y conocimientos las innovaciones técnico-científicas de su aparato productivo.

El jurisconsulto Francisco Javier de Gamboa (1717-1794) fue quien planteó que era necesaria una revisión del estado en que se hallaba la industria minera de la Nueva España. En 1774 los propietarios de minas de la Nueva España solicitaran, para su mejor organización, que se creará un Tribunal de Minería, y se fundará en la ciudad de México un Colegio o Seminario Metálico con el propósito de preparar individuos para el laboreo de las minas, así como el beneficio de los metales (SALDAÑA, 1996, pp. 151-202). Desde España el rey Carlos III trazó un vasto plan para llevar técnicos y expertos formados en la Escuela de Minas de Friburgo para que promovieran la minería en la Nueva España, y por Real Cedula de 1 de julio de 1776 se ordenó la erección en México de un Real Tribunal General de la Minería, autorizado para crear un banco de avíos cuyos fondos deberían ser aplicados al fomento de las minas y el sostenimiento de un Colegio Metálico (RAMÍREZ, 1890, pp. 20-79; FLORES, 2000, pp. 21-57). Éste fue creado el $1^{\circ}$ o de enero de 1792, y su primer director, el logroñes Fausto de Elhuyar (1755-1833), reconoció que los problemas de que adolecía la minería de la Nueva España se debían, en parte, al escaso conocimiento geológico del territorio novohispano; al insuficiente desarrollo de técnicas específicas para la prospección, explotación y beneficio de los metales y a la débil o inexistente reflexión científica sobre las características de los yacimientos minerales y su entorno geológico, lo que redundaba en un pobre aprovechamiento metalúrgico de sus menas (GALVEZ, 1933). El puntual e inteligente señalamiento de Elhuyar, abrió las puertas a una reforma de la minería y la metalurgia en el ocaso del imperio español y a un proceso de institucionalización de las nuevas disciplinas: la mineralogía y la geología (CAÑERO Y ALZOLA, 1933, pp. 379-629; PESET, 1987, pp. 143-267; CASTILLO, 2005; PELAYO Y REBOK, 2002-2003, pp. 67-90).

\section{ANDRÉS DEL RÍO (1764-1849), TRANSICIÓN DEL NEPTUNISMO AL PLUTONISMO}

Andrés del Río fue uno de esos hombres de ciencia que llegaron a Nueva España en los últimos años del siglo XVIII, contratado por el gobierno español para formar funcionarios mineros calificados en las artes de los metales; impulsar la investigación sobre los recursos mineros y minerales; y promover en los reales de minas del virreinato innovaciones tecnológicas que asegurasen la buena marcha de las explotaciones mineras y las finanzas del reino. Había hecho sus estudios científicos por espacio de 12 años en instituciones europeas de gran prestigio, como la Real Academia de Minas de Almadén, España (1782-1783); l'Ecole Royale des Mines, Francia (1785-1786); Collège de Fran- 
ce, Francia (1786); Bergakademie de Freiberg, Alemania (1787-1789); Real Academia de Minas y Bosques, Hungría (1790); Laboratorio del Arsenal, Francia (1793-1794), entre otras. En esa "comunidad científica" internacional, aún pequeña, destacan los nombres de algunos de sus profesores y colegas: Christophe Störr, Jean D'Arcet, Abraham Gottlob Werner, Antón von Rupprecht, Leopold von Buch; Laurent Lavoisier; Just Haüy, Dieudonné Dolomieu, Alejandro de Humboltd, Benedic Saussure y Luís Lindner, entre otros, todos de gran significado internacional en el desarrollo de las ciencias y los saberes en la primera mitad del siglo XIX (URIBE Y CORTÉS, 2006, pp. 491-518).

En el Real Seminario de Minería de la ciudad de México impartiría su cátedra de mineralogía ininterrumpidamente de 1795 hasta 1805, y la de docimasia y ensayos entre 1798 y 1805 . Después, con algunas interrupciones, cumplió con su labor docente entre 1814 y 1847, fecha ésta última en que inicia su proceso de jubilación. En ese tiempo compaginó la docencia con sus trabajos de investigación, las traducciones de manuales y tratados sobre Orictognosia, Geognosia, Geometría Subterránea y Geología, y el indispensable ejercicio de exploración y trabajo de campo que lo llevaría a descubrir nuevos minerales y sustancias como el "Erythronium" o elemento 23 de la tabla periódica (BARGALLÓ, 1966, pp. 11-79; RUBINOVICH, 1992, pp. 03-70; URIBE, 2008, pp. 147-164). También cumplió con distintas comisiones oficiales como el establecimiento de la primera ferrería industrial en la América española, ubicada en la región de Coalcomán (1805-1809), el asesoramiento técnico a los empresarios poblanos de la industria de cerámica (década de 1830), cumpliendo así con los objetivos para los que había sido contratado (RAMÍREZ, 1890, pp. 310-320; URIBE, 2006, pp. 231-260; URIBE y CORTÉS, 2006, pp. 491-518).

Aquí intentamos esbozar algunos elementos exploratorios de las prácticas discursivas del conocimiento geológico en la obra de Andrés del Río a partir del reconocimiento de las condiciones de emergencia del saber, el discurso y las reglas de formación de los objetos geológicos. De manera individual confluyen en él tres estructuras de pensamiento que, a la vez, se constituyen en herramientas o dispositivos para organizar sus hallazgos como científico; de un lado, un pensamiento dirigido hacia la observación de la naturaleza; un pensamiento geométrico que necesariamente se constituiría en una herramienta de interpretación en sus hallazgos, y un pensamiento filosófico crítico.

En su primera época profesional mantuvo un reconocimiento a Carl Linné, Louis Leclerc, Georges Cuvier y Abraham Gottlob Werner, de los que aprendió sus teorías y procedimientos para desentrañar los secretos de la naturaleza. De Linné aprendió la "sistemática" que daría lugar al nacimiento de la paleontología; de Leclerc, la sucesión de fauna y flora junto con las alternancias de emergimientos y sumergimientos de los continentes a lo largo de la historia de la Tierra; de Cuvier, el estudio de los restos de animales fósiles con el fin de demostrar que eran distintos a los actuales, cuya desaparición las atribuyó a terribles "cataclismos" geológicos; y de su maestro Werner, creador de la teoría neptunista, que la Tierra fue en su origen un núcleo sólido cubierto por un océano que actuó como verdadero agente del cambio geológico (GÍO-ARGÁEZ y RODRÍGUEZ, 2003, p. 86).

En sus estudios de Paleontología, Geognosia, Física, Química y Matemáticas en Europa, seguramente conoció la obra de René Descartes (1596-1650), y en Alemania la de 
Immanuel Kant (1724-1804). Así las cosas, Descartes era considerado el creador del mecanicismo y de la geometría analítica. En la obra de Del Río, en sus descripciones minuciosas de rocas y minerales, se puede observar uno de los principios cartesianos que sostenía que la única posibilidad de conocer la realidad física era reduciendo los fenómenos a sus partes, y después era necesario y suficiente analizar la interacción de las partes para explicar esos fenómenos. "En las ciencias experimentales, decía Andrés del Río, la falta de observación no se remplaza con adivinar”. Buena parte del trabajo de investigación que desarrolló Del Río, tenía que ver con esa concepción filosófica. Para ello, dice: "sirven muy bien los caracteres de Wérner que juntos con las análisis químicas prestan fundamento para una clasificación más universal, que abraza mas objetos, y bastante sistemática sin necesidad de tantos apéndice" (DEL RÍO, 1820-1821, pp. 246-257). Apelaba con ello a una reflexión universal de los descubrimientos locales.

La recepción del pensamiento kanatiano en la obra de Andrés del Río, parece tan evidente como la primera. En su estancia en Freiburg, en 1787, la trayectoria académica de Kant era ya prominente. Para 1787 había publicado éste obras tan importantes como la Crítica de la razón pura, los Prolegómenos a toda metafísica del porvenir, la Crítica de la razón práctica y la Fundamentación de la metafísica de las costumbres. La ética que practicó Del Río en vida tienen mucho en común con lo que planteó Kant. En la Metafísica de las costumbres (1797) Kant describió su sistema ético, basado en la idea de que la razón es la autoridad última de la moral. Describió dos tipos de órdenes dadas por la razón: el imperativo hipotético, que dispone un curso dado de acción para lograr un fin específico; y el imperativo categórico, que dicta una trayectoria de actuación que debe ser seguida por su exactitud y necesidad. Para Kan el imperativo categórico era la base de la moral, premisa que fue resumido por Del Río en estas palabras claves: "No todos podemos aspirar a la celebridad vinculada en un mérito del primer orden; pero todos debemos aspirar a la reputación de ciudadanos útiles, cada uno según sus alcances” (DEL RÍO, 1803, p. 419).

En oposición a la época clásica que se las ingenió para ver lo menos posible y restringir el campo de la experiencia, Andrés del Río "vio" objetos que no estaban ahí, los comprendió, los nombró y les dio un estatuto, entre ellos su famoso descubrimiento del "eritronio" (1801), conocido a partir de 1830 como Vanadio en la Tabla Periódica. También integró una serie de principios que permitieron armar sistemas clasificatorios para la enseñanza de la mineralogía y la geología en el Real Seminario de Minería y después Escuela Nacional de Minería. Y los formuló con el rigor que le otorgaban las matemáticas y la filosofía crítica: "Yo bien sé con Kant - decía Andrés del Río en 1820 -, y estoy convencido de que en estas ciencias no hay mas que la parte matemática que sea verdaderamente científica" (DEL RÍO, 1820-1821, pp. 173-182, 255). Científica no en el sentido determinista, sino en la búsqueda de una alta probabilidad entre saber y verdad. Ese principio define su "obra".

La biografía de Andrés del Río representa un excelente espacio de análisis, pues transita el gran debate entre Neptunistas y Plutonistas. Vivió el desarrollo de la orictognosia o mineralogía y de la geognosia consagrada al estudio de la formación de las masas pétreas por acción del fuego interno - como se decía antes - o de fenómenos acuosos. Ese período (más o menos de 1780 a 1840) se caracteriza también por la aparición de 
las primeras obras centradas en cuestiones geológicas (mineralógicas, paleontológicas, cristalograficas y estatigráficas). Del Río escribió alrededor de 60 trabajos entre artículos, reportes técnicos y monografías científicas sobre distintos fenómenos de la corteza terrestre. Pero fueron quizá sus trabajos: Elementos de Orictognesia o del conocimiento de los fósiles (1795); Mineralogía. Carta dirigida al señor Abate Hauy (1820-1821); Analyse des deus nouvelles espéces minérales composées de séléniure de zinc et de sulfure de mercure (1829); Elementos de Orictognesia, o del conocimiento de los fósiles según el sistema de Bercelio (1832); Manual de Geología extractada de la Lethaea geognostica de Bronn con animales y vegetales perdidos o que ya no existen, mas característicos de cada roca, y con algunas aplicaciones a los criaderos de esta República (1841), los que mejor ejemplifican el parteaguas entre el naturalismo y la geología moderna. A esto Michael Foucault lo denomina constitución del saber acerca de la tierra como cuerpo de conocimiento especializado y delinea la llegada o el nacimiento de la geología como una ciencia, en tanto sistema discursivo y práctica científica (FOUCAULT, 1966, 1979, 1992).

En ese tiempo, Andrés del Río intentó desde Nueva España-México proporcionar una nomenclatura propia para la mineralogía en el seno de la lengua española, aunque no triunfaron sus consejos porque quizás no hubo seguidores en este dominio lingüístico que defendieron su modelo. Entre 1794 y 1848, Del Río debatió los adelantos de estas disciplinas con sus pares de Europa y Estados Unidos, e incorporó a la discusión sus propios hallazgos y descubrimientos, junto con las aportaciones de sus colegas mexicanos, como evidencias de una cierta evolución uniforme del globo terráqueo. Al mismo tiempo sometió a duras críticas el posicionamiento de James Dwight Dana (1813-1895), el ya afamado geólogo estadounidense, con respecto a la nomenclatura anglosajona que terminaría imponiéndose tiempo después en la enseñanza de la mineralogía y geología como expresión indiscutible de la hegemonía científica que alcanzaría en el mundo occidental (DEL CASTILLO, 1864, p. X). En su discurso geológico de 1840, Del Río asienta: "Nos ha regalado el mineralogista Dana una nomenclatura inútil, impropia, y sobre todo bárbara... y esto se llama simplificar la ciencia" (DEL RÍO, 1848, p. 2).

Frente a la avasalladora cultura estadounidense, sentenció: "yo escribo para el futuro en la confianza de que al cabo ha de triunfar la razón también contra este despotismo" (DEL RÍO, 1848, p. 257). Pero lo cierto es que ese discurso se alejó de los modelos alemanes y franceses a los que él estaba más próximo para sucumbir ante las obras estadounidenses que se tradujeron prolijamente a mediados del siglo XIX y de las que nos llegó la mayor parte de este léxico especializado.

Lector atento y sistemático de la producción científica de su tiempo, conoció la obra de Charles Leyl ${ }^{(1)}$ en su estancia en Filadelfia de 1829 a 1835, y su famosa tesis: las mismas fuerzas geológicas que modificaron la Tierra en la prehistoria, se hallan hoy y seguirán siempre activas. En su Manual de Geología, que se publicó en 1841, Del Río evidenció su posicionamiento respecto al paradigma establecido por Charles Lyell en Principios

(1) Lyell, Charles (Kinnordy, Escocia, Gran Bretaña, 1797-Londres, 1875) Geólogo escocés. Basándose en diversos trabajos del geólogo James Hutton, desarrolló la teoría de la uniformidad, que establecía que los procesos naturales que cambian la Tierra en el presente son los mismos que actuaron en el pasado. 
de la Geología, para explicar las transformaciones ocurridas en el globo terráqueo a lo largo del tiempo histórico. En la nota introductora a su Manual, asentaba que "las pocas adicciones que se deben hacer a mi Manual de Geología", ya estaban "contenidas en los elementos de la misma del año pasado de Mr. Lyell, considerando las formaciones de abajo arriba" (DEL RÍO, 1843, p. 2), es decir, las modificaciones habidas en la estructura interna del planeta y los cambios ocurridos en la superficie de la corteza terrestre. Con ello, Andrés del Río asumía al final de su vida productiva una concepción geológica de la tierra distinta a las enseñanzas catastrofistas de su maestro Werner (BARGALLÓ, 1964).

Sin duda sus planteamientos en la vertiente conceptual sentaron la base de diversas disciplinas que se cultivarían con profusión en México en los siguientes cien años (1850-1950) y que hoy constituyen la geología. Esas redes conceptuales constituyeron verdaderos cimientos de la práctica científica en la época. A manera de hipótesis se enumera apretadamente las redes conceptuales que concibió y formuló Del Río, así como las disciplinas que cultivo: 1) la ubicación espacial de los minerales y la Geología geográfica; 2) el estudios de los cristales y la Cristalografía; 3 ) los estratos de la tierra y la Estratigrafía; 4) la formación de fósiles y la Paleontología.

Asume con presteza los nuevos derroteros de la ciencia geológica, y se convierte en el promotor de su incorporación, como materia y disciplina, al plan de estudios en la Escuela de Minería, propuesta que no alcanzó a ver cristalizada en vida, ya que el gobierno negó los recursos económicos para tal propósito (DEL RÍO, 1843, p. 8). Andrés del Río fue parco al realizar la crítica a las decisiones gubernamentales que en 1833 suprimieron la reforma educativa que modernizaba los contenidos y orientación de los estudios profesionales que se impartían en la Escuela de Minería; a la decisión de los funcionarios públicos del ramo de no dotar de fondos a la cátedra de Geología; o a la discontinuidad visible en la formación de alumnos y pasantes en la Historia Natural, que "junto con los profesores, eran el alma del Colegio". Apesadumbrado por las restricciones gubernamentales al trabajo científico de los profesores, concluía: "Nosotros trabajamos mas; pero escribimos en arena por falta de repasos ó de la argamasa que conglutinaba los granos de arena para conservar las impresiones" (DEL RÍO, 1843, p. 8; DEL RÍO, 1848, p. 4).

Como profesor titular de la cátedra de mineralogía en el Real Seminario de Minería, después Colegio de Minería, formó en sus aulas a un número importante de profesionales de la talla de Casimiro Chovell, Francisco Álvarez, José Joaquín de Zárate, Vicente Herrera, José Antilla, Manuel Cotero, Manuel Cueto, Félix Rodríguez, Vicente Valencia, José Oteiza, Sixtos Cardona, Rafael Cardoso, Juan Arezorena, Manuel Ruiz de Tejada, José María de Bustamante, Ignacio Alcocer, Sebastián Segura, Joaquín Velásquez de León, Lucas Alamán, Blas Barcárcel, Antonio del Castillo, Francisco Díaz Covarrubias, Manuel Riveras Cambas, Manuel Fernández Leal (RAMÍREZ, 1890, pp. 135-195)(2), entre otros, que contribuirían decisivamente al desarrollo y consolidación de la mineralogía y de la geología mexicana en la segunda mitad del siglo XIX (URIBE, 2007). Con Andrés del Río se abre y se cierra una etapa de trabajo científico en México, y se fijan las bases para

(2) Memoria presentada a S. M. el Emperador por el Ministro de Fomento Luís Robles Pezuela de los trabajos ejecutados en su ramo en el año de 1865, México, Imprenta de J. M. Andrade y F. Escalante, 1866, p. 359-360. 
el desarrollo de las modernas disciplinas, pues a decir de él mismo, en la postrimería de su vida, "cada descubrimiento nos indica que queda infinitamente mas que descubrir, y nos muestra la intima relación de estas ciencias entre sí y con las artes” (DEL RÍO, 1843, p. 8) (3).

\section{ANTONIO DEL CASTILLO (1820-1895), INSTITUCIONALIZACIÓN DE LA CIENCIA}

Antonio del Castillo fue el alumno predilecto de Andrés del Río, que se graduó de ingeniero de minas en 1845. Entre 1846 y 1848 fungió como Secretario del Colegio de Minería. Fue nombrado sustituto de Andrés del Río en la cátedra de mineralogía en 1847; y en 1851 se convirtió en titular de la misma por concurso de oposición (DÍAZ Y OVANDO, 1998, p. 1.477-1.486) ${ }^{(4)}$. Desde un primer momento introdujo importantes cambios en los contenidos de su cátedra y renovó la práctica científica de la misma. En el ámbito epistemológico realizó precisiones sustantivas de gran significación para el futuro de la geología, suscribiendo los nuevos paradigmas postulados por Charles Lyell en sus Principios de Geología, como "la ciencia que investiga los sucesivos cambios que han tenido lugar en los reinos orgánico e inorgánico de la naturaleza, que investiga las causas de estos cambios y la influencia que han ejercido en la modificación de la superficie y la estructura externa de nuestro planeta" (LYELL, 1830, p. 01).

En el ámbito de la práctica científica de la disciplina, pugnó por la ejecución de un programa de reconocimiento pormenorizado del territorio nacional que aportara la información y los hechos que dieran sustento a nuevas explicaciones científicas de los fenómenos geológicos del territorio mexicano. Apoyó diversas iniciativas, como la creación en 1853 del Colegio Nacional de Agricultura, en cuyos planes de estudio se incorporaron las materias de orictognocia y geología, con un valor espistemológico semejante a la química, la física o la botánica (DUBLAN Y LOZANO, 1876-1911, tomo VI, documento 4001, 1851-1853); también pugnó por la nacionalización de los saberes sustrayendo de la ciudad de México el monopolio del ethos científico. Su primera experiencia en esta dirección fue su participación en la creación de la Escuela Práctica de Minas de Fresnillo, en Zacatecas, en 1853, en la cual se desempeñaría como profesor de Laboreo y Mecánica aplicada a las Minas (AGUILERA, 1896, pp. 03-06).

A partir de esa fecha, Del Castillo mantuvo una posición destacada en la comunidad científica mexicana. Cuando el ejercito napoleónico invadio México en 1862, trabajó con los franceses en la Comisión Scientifique du Méxique encargada de los estudios sobre geografía, geología, mineralógica, botánica, zoología y meteorología del país, que le valió el reconocimiento de "primer geólogo mexicano del imperio". Su experiencia científica

(3) Andrés del Río vivió los últimos años de su vida, después de su regreso de Estados Unidos, en una franca situación de desventaja respecto de la que vivían sus colegas alemanes, franceses y estadounidenses, con los que de manera abierta mantuvo una lucha frenética en la definición de una nomenclatura moderna para la ciencia mineralógica. A su regreso del "exilio", encontró una Escuela de Minería sin rumbo y con escaso apoyo gubernamental; una comunidad científica que si bien reagrupaba sus energías e intereses en la Sociedad Mexicana de Geografía y Estadística (1833), contaba con muy pocos profesionistas en las ciencias mineralógica y geológica con los cuales dealogar.

(4) Archivo General de la Nación (AGN). Galería 5: Justicia e Instrucción Pública, vol. 38, exp. 44, fs. 332-347. 
al lado de los expedicionarios franceses, entre los que destacaron los mineralogistas y geólogos E. Guillemin-Tarayre, Aguste Dollfus, E. de Montserrat y P. Pavie, se traduciría en una serie de artículos sobre la mineralogía y la meteorología mexicana, áreas disciplinares que cultivaría a lo largo de su vida (MORELOS, 2012; AZUELA, 1995, pp. 103-105), y en una propuesta metodológica para concluir la construcción de la primera carta geológica de México que la Comisión Scientifique du Méxique dejara inconclusa al triunfo de las fuerzas republicanas sobre el ejército imperial de Maximiliano de Austria. La experiencia que capitalizara Del Castillo, al lado de otros colegas mexicanos entre los que vale la pena mencionar a Antonio García Cubas, Francisco Jiménez, Manuel Orozco y Berra, Leopoldo Río de la Loza o Ramón Almarez, sería de gran trascendencia para el desarrollo posterior de la geología en México, pues "simbolizaba la difusión de la metodología de frontera para la investigación geológica” (AZUELA, 2005).

A partir de 1867, con la restauración de la República, la Escuela Imperial de Minas en el gobierno de Maximiliano es convertida en Escuela de Ingenieros. Ese mismo año Antonio del Castillo retoma su cátedra de mineralogía y se involucra en el diseño de las nuevas políticas sobre educación y ciencia que promueve el presidente Benito Juárez (CUEVAS, 2002, pp. 40-41). Mejor posesionado en el mundo científico mexicano, consideraba que la enseñanza de la mineralogía y la geología debían sustentarse en una práctica sistemática de exploración, recopilación de muestras, análisis de laboratorio, formación de colecciones e interpretación de los hechos científicos de conformidad con los paradigmas científicos en boga. A pesar que tenía la formación de ingeniero de minas, en sus enseñanzas y actividades en general puso mayor énfasis en la paleontología, estratigrafía y geología de campo, llevando frecuentemente a sus alumnos en excursiones geológicas a diferentes partes de la República, recolectando material para la primera carta geológica de México (CARRERA, 1956, p. 08; MORELOS, 2013). El primer registro oficial de fósiles mexicanos lo realiza Antonio del Castillo en 1869, quien clasifica algunos mamíferos fósiles localizados en el Vallle de México (DEL CASTILLO, 1869, pp. 479-482; DEL CASTILLO, 1879, pp. 484-485) ${ }^{(5)}$.

A principios de los años de la década de 1870 Antonio del Castillo es nombrado director de la Escuela de Ingenieros, después de haber cubierto un periodo corto como subdirector de la misma, desde donde promueve una reforma al plan de estudios que aprobó el Congrego en 1877 (RAMÍREZ, 1890, p. 11). El plan de estudios anterior, de 1867, ponía el acento en la formación teórica, y reducía al mínimo el trabajo de campo. Las modificaciones introducidas por Del Castillo buscaron equilibrar la parte teórica con las prácticas de campo, para lo cual los estudiantes deberían concluir su formación iniciada en la Escuela de Ingenieros en alguna Escuela Práctica de Minas, que debería establecerse para tal efecto (DUBLAN y LOZANO, 1876-1911, tomo XIII, pp. 130-131).

Para ello propuso, y se aprobó, una asignatura que con el nombre de "Mineralogía, geología y paleontología" que articularía la enseñanza de la geología en el ámbito

(5) Sin embargo, no es hasta algunos años después que Bárcenas, al describir un crustáceo y después los fósiles característicos de las rocas mesozoicas de México, registra por primera vez los invertebrados fósiles del país. Mariano Bárcena, "Descripción de un crustáceo fósil del género Spheroma (S. burkartii) y reseña geológica del Valle de Ameca, Jalisco", La Naturaleza, 3, 1875, p. 355-361; Mariano Bárcena, "Datos para el estudio de las rocas mesozoicas de México y sus fósiles característicos", Sociedad Mexicana Geografía Estadística, 3, 2, 1875, p. 369-405. 
académico, y de la que sería su titular hasta poco antes de su muerte en 1895. "Para el devenir de la geología, Antonio del Castillo logró un cambio apenas perceptible a los ojos del neófito: atar los contenidos cognoscitivos de la geología en una solo asignatura denominada 'Mineralogía, geología y paleontología'. Y de paso consolidó el monopolio de la enseñanza de la disciplina en el ámbito académico" (AZUELA, 2005, p. 190 y ss.).

La reforma curricular al plan de estudios llevada acabo por Antonio del Castillo contemplaba igualmente la apertura de un sistema nacional de Escuelas Prácticas de Minas en donde los alumnos de la Escuela Nacional de Ingenieros pudieran realizar sus practicas de campo como parte de su formación profesional. Desde luego, aprovechó las presiones del gremio minero para sustentar y legitimar la apertura de la Escuela Práctica de Minas de Pachuca, de gran significado en el proceso de institucionalización de la geología y nacionalización de los conocimientos geológicos, sustrayendo el monopolio cognoscitivo a las instituciones de educación radicadas en la ciudad de México.

Vinculado al grupo en el poder, años después, el 6 de enero de 1881 Antonio del Castillo es nombrando nuevamente director de la Escuela Nacional de Ingenieros en sustitución del ingeniero Manuel Fernández Leal (AGUILERA, 1896, pp. 04-06; CARRERA, 1956, pp. 04-06; DÍAZ Y DE OVANDO, 1998, tomo III, p. 2657). A partir de entonces inicia una intensa y larga negociación con las autoridades del gobierno federal con el objeto tanto de diversificar las opciones educativas, con la apertura de nuevas especialidades en ingeniería (PALACIOS, 1911, pp. 03-15; URIBE, 2001), y, al mismo tiempo, refundar los estudios geológicos con plena autonomía espistemológica de otras áreas, profesiones y actividades. Promueve y consigue financiamiento adicional para modernizar los laboratorios, enriquecer la biblioteca con nueva literatura y ampliar y sistematizar las colecciones mineralógicas propiedad de la institución.

A éste ingeniero de mians se debe, en gran medida, la independencia de la geología, como disciplina, de las actividades propiamente mineras, a las que había estado supeditada desde su nacimiento, y también la legitimidad social de ese conocimiento como baluarte para la cientifización de otras actividades como la agrícola o la hidráulica (GALINDO Y VILLA, 1903, p. III-V; AGUILERA, 1904, pp. 11-29).

Ahora bien, con los cambios en las políticas educativas después de 1867, los programas de estudios se diversificaron en la mayoría de las instituciones de educación. Fuera de la ciudad de México, el Instituto de Toluca, capital del púgnate Estado de México, incorporó las carreras de geógrafo e hidrógrafo, ensayador de metales y las de ingeniero topógrafo, mecánico, civil y de minas (CASTAÑEDA, 1995). Lo mismo sucedió en Guanajuato, San Luís Potosí, Aguascalientes, Nuevo León, Puebla o Querétaro, y otros estados, en donde existían explotaciones mineras, trabajos de infraestructura en comunicaciones, y pugante actividad industrial que demandaban personal capacitado en las ciencias y técnicas modernas. En Guanajuato, con la reforma a la ley local de educación en 1870, se crearon las carreras de ingeniero en minas, geógrafo y topógrafo, y se actualizaron las tradicionales de beneficiador de metales y ensayador.

En Zacatecas, el Instituto Científico y Literario fue representativo de la nueva orientación en los programas de estudio a nivel nacional: de la enseñanza de la lógica, la metafísica y ética, de corte escolástica y diseochesca, se pasó a la enseñanza de la aritmé- 
tica, álgebra, geometría, trigonometría, dinámica, hidrodinámica, óptica, calculo infinitesimal y química práctica. En 1867 se incorporan las carreras de ingeniero de minas, ensayador de metales y otras, que debió dirigir Ignacio Herrera, cirujano, farmacéutico, ingeniero de minas y ensayador de metales titulado en el antiguo Colegio Nacional de Minas, y fundador tanto de la Escuela Práctica de Minas de Fresnillo en 1860, como de la Escuela Nacional de Ingenieros en la ciudad de México años después (ROBLES, 2001).

Siguiendo con la tradición inaugurada por José Antonio de Alzate y Ramírez, considerado como el primer periodista científico de la Nueva España, los naturalistas, geógrafos y geólogos como Andrés del Río, José Gómez de la Cortina, Manuel de Herrera, Manuel Ruiz de Tejeda, Tomás Ramón del Moral, Francisco Ortega, Juan Orbegozo, Manuel Bustamante, Joaquín Vázquez de León, Santiago Ramírez, Mariano Bárcena o el propio Antonio del Castillo se organizaron en redes y comenzaron a publicar revistas, periódicos, manuales y libros destinados a difundir sus hallazgos científicos y a promover la incorporación de sus contenidos en los programas de enseñanza, ahí donde la mineralogía, la geología o la paleontología habían obtenido un estatus epistemológico al lado de otras disciplinas científicas.

Tres de las más importantes asociaciones científicas con sus respectivos órganos de difusión fueron la Sociedad Mexicana de Geografía y Estadística, 1833, y su Boletín con el mismo nombre (1839); la Sociedad Mexicana de Historia Natural, 1870, y La Naturaleza (1870), que era su periódico científico (CARPY, 1986, pp. 19-250); y, por último, la Sociedad Científica "Antonio Alzate", 1884, y sus Memorias y Revista (1887). No son las únicas pero si quizá las de mayor relevancia sin las cuales no se entendería a cabalidad los procesos de institucionalización y profesionalización de las ciencias de la Tierra en México. En ellas figura la geología como disciplinas y en cuyas páginas aparecieron numerosos artículos sobre geología, mineralogía, paleontolgía, geoquímica, etc. (BELTRÁN, 1968, pp. 119-120; AZUELA, 1996, pp. 39-118; CUEVAS, 2002, pp. 67-68; MORELOS, 2012; URIBE, 2013, pp. 117-142), que contribuirían a su autonomía epistemológica en la segunda mitad del siglo XIX.

También durante la segunda mitad del siglo XIX se comenzó a publicar tratados, artículos, informes y notas pensadas y formuladas para enseñar las disciplinas de conformidad con los programas de estudio del sistema educativo nacional. Mariano Bárcena (1842-1899), naturalista y geólogo, publicó numerosos artículos sobre la geología de los estados de Aguascalientes, Hidalgo y su estado natal, Jalisco, incluyendo el primer mapa geológico de Guadalajara y región circundante. Sus contribuciones más importantes fueron: Datos para el estudio de las rocas mesozoicas de México y sus fósisles característicos (BARCENA Y DEL CASTILLO, 1875) y Materiales para la formación de una obra de paleontología mexicana, publicada en 1877, que constituyen el comienzo de investigaciones estratigráficas y paleontológicas en México hechas por mexicanos. Publicó también estudios sobre temblores en Jalisco, sobre los volcanes el Ceboruco y de Colima y sobre las obsidianas de México. Fue profesor de geología en la Escuela Nacional de Ingenieros, donde utilizó su libro Tratado de Geología, como texto, que fue publicado en México, en 1886, y fue fundador y director del Observatorio Meteorológico Central de México, establecido en 1877 (DE CSERNA, 1990, p. 911). 
Otro de los grandes impulsores de los estudios geológicos en México y de su profesionalización cognoscitiva fue el ingeniero de minas Santiago Ramírez (1841-1922). La orientación científica de Ramírez dentro de la geología fue más hacia una ciencia utilitaria: los yacimientos minerales, pero publicó numerosos artículos sobre la geología de éstos y su distribución, sobre yacimientos de carbón y un libro intitulado Litología; introducción al estudio de las rocas, que se editó en 1886 (AGUILERA, 1896, p. 62).

En Zacatecas, el Instituto Científico y Literario, editó en 1905 los trabajos de José Árbol Bonilla: Nociones de mineralogía; Nociones de geología; Nociones de meteorología y climatología, y su Programa de clase de cosmografía con prácticas en el observatorio, los cuatro al inicio del ciclo escolar 1905 (ÁRBOL Y BONILLA, 1905 a, b, c, d). Y cinco años después, en 1910, la obra Compendio de mineralogía de Lorenzo T. Villaseñor, adaptada para texto de la clase de química y nociones de mineralogía que en ella se cursaba (VILLASEÑOR, 1910). En cada entidad federativa del país se habían escrito obras fundamentales sobre mineralogía, paleontología y geología, que junto con las de Antonio del Castillo, Santiago Ramírez, Mariano Bárcena y José G. Aguilera, y otros, eran incorporadas a su lectura obligada en los programas de estudio en donde se impartían dichas materias (AGUILAR Y SANTILLÁN, 1898, pp. 04-93).

Desde luego, la profesionalización de las Ciencias de la Tierra en México contó con la experiencia y los conocimientos producidos por distintas comisiones oficiales creadas temporalmente para asesorar al grupo gobernante en la elaboración de las políticas públicas y en la toma de decisiones para el fomento industrial. Entre las comisiones temporales de mayor importancia podemos destacara la Comisión Geológica del Estado de México, que fue el primer servicio geológico estatal de la República Mexicana; la Comisión Geográfico-Exploradora de México que laboró de 1879 a 1884, y que tenía como fin principal el reconocimiento del territorio nacional y sus recursos, así como el levantamiento de la carta geográfica de la República; y finalmente la Comisión Científica de Sonora de 1886 (AGUILERA, 1896, p. 62; BAZANT, 1984, pp. 254-297).

A partir del año de 1881 Antonio del Castillo, ya en funciones en su segundo periodo como director de la Escuela Nacional de Ingenieros emprendería las gestiones ante el gobierno federal parea crear el Instituto Geológico, institución que debería encargarse de realizar la enseñanza y la investigación de la geología de manera sistemática. El 26 de marzo del 1886 elevó al gobierno federal el proyecto de creación de un organismo oficial que se encargara de sistematizar la ya extensa literatura geológica existente y promover con el rigor científico moderno la investigación geológica del territorio nacional (CARRERA, 1956, pp. 04-06; AZUELA y GUEVARA, 1998, pp. 91-93). El primer paso fue crear en marzo de 1888 una Comisión Geológica (AGUILERA, 1896, pp. 81-82). Dicho organismo quedó bajo la dirección de Antonio del Castillo, cuya tarea fue la elaboración de un bosquejo geológico de México, junto con la carta geológica y minera del país (AGUILAR y SANTILLÁN, 1898, pp. 04-93). Entre las decisiones tomadas por Del Castillo al frente del nuevo organismo, destaca la conformación de las primeras colecciones científicas en paleontología, en las que integró sus propios materiales y hallazgos resultado de mas de treinta años de investigación. Dispuso también "la organización y arreglo" de los registros de vertebrados fósiles publicados por Richard Owen (1869), así como 
los de los primeros invertebrados descritos por el geólogo jalisciense Mariano Bárcena (1875) y los fósiles de mamíferos listados por el propio Del Castillo (1869, 1879). "En esta colección, también fueron alojados materiales extranjeros, principalmente de Europa, así como material recolectados y adquiridos (posteriormente) por los investigadores del Instituto" (CARREÑO y MONTELLANO-BALLESTEROS, 2005, pp. 137-147).

La Comisión Geológica fue sustituida el 17 de diciembre del mismo año con la creación del Instituto Geológico Nacional ${ }^{(6)}$. Entre 1888 y 1891 el Instituto Geológico Nacional desarrolló su trabajo con un reducido personal y un exiguo presupuesto. No obstante, los primeros resultados de investigación comenzaron a publicarse al año siguiente ${ }^{(7)}$. El más importante de estos fue la primera edición de la Carta Geológica y Minera de México escala 1:3`000,000, de la autoría de Antonio del Castillo (1889). Al crearse el Instituto de Geología, Antonio del Castillo fue nombrado director, puesto que ocupó hasta julio de 1895, poca nates de su muerte. Su lugar es ocupado por su discípulo el ingeniero José Guadalupe Aguilera, subdirector del Instituto y lugarteniente del Castillo desde la época de la Comisión Geológica (CARREÑO, 1938, p. 124; MORELOS, 2012).

\section{JOSÉ G. AGUILERA (1857-1941), PROFESIONALIZACIÓN E INTERNACIONALIZACIÓN DE LAS CIENCIAS DE LA TIERRA}

José G. Aguilera había nacido el 5 de febrero de 1857 en Mapimí, Durango. Sus primeros estudios los realiza en su pueblo natal, y más tarde en el Instituto Juárez de Durango en el que llega a desempeñar el cargo de Prefecto del Instituto. A los 19 años se radica en la ciudad de México para hacer estudios de preparatoria, al término de los cuales se inscribe en la Escuela de Minería en la que cursa la carrera de ensayador, apartador y beneficiador de minerales entre 1877 y 1880. En 1879, un año antes de terminar la carrera de ingeniero de minas, es nombrado Especialista de Análisis Químicos, y encargado de los Gabinetes de Mineralogía y Geología, puesto que conserva hasta 1882 en que comienza a colaborar como ingeniero geólogo en la Comisión Geográfico Exploradora, fundada en 1878. A la edad de 25 años recorre los estados de Puebla, Oaxaca y Tlaxcala durante dos años. Después se le comisiona por el gobierno de Porfirio Díaz como delegado de México a la Exposición Universal que tuvo lugar en Nueva Orleáns, en donde presenta sus trabajos de investigación y sus colecciones de minerales, rocas y fósiles.

De Nueva Orleáns, José G. Aguilera viaja a Washington y se inscribe en el Instituto Smithsoniano, en el que estudia dos años, de 1884 a 1886. En 1886 regresa a México a hacerse cargo de la Comisión Científica de Sonora, como geólogo en jefe de la misma, para estudiar el temblor de Babispe, de 3 de mayo de 1887, que produjo una falla de 81 kilómetros de largo y causo muerte y destrucción en todos los poblados cercanos al epicentro. Su informe Estudios de los fenómenos Sísmicos del 3 de mayo de 1887 (AGUI-

(6) Diario Oficial, tomo XIX, n. 152, México, 25 de diciembre de 1888, p. 02.

(7) "Lamentablemente, no se incluyó en esta tarea el levantamiento geológico sistemático del país, en el que se hubiera podido utilizar como base las hojas topográficas de la Comisión Geográfico-Exploradora, y se omitió así la creación de un soporte real para cualquier investigación geológica, carencia que aún existe en los albores del siglo XXI”. De Cserna, 1990, p. 11. 
LERA, 1887, p. 07) ${ }^{(8)}$, fue considerado "el primer trabajo científico que se ha llevado a término en México acerca de los fenómenos sísmicos” (PALACIOS, 1911, pp. 06-18; CARREÑ , 1938, p. 123). Al concluir ese trabajo se incorporó a la Comisión Geológica encargada de elaborar la Carta Geológica de la República Mexicana, formada y dirigida por el ingeniero Antonio del Castillo, director de la Escuela Nacional de Ingenieros y promotor del Instituto de Geología.

A la muerte de su mentor, José G. Aguilera asume la dirección del Instituto Geológico Nacional, e inicia el recorrido final de la profesionalización de la geología mexicana. Siguiendo los fundamentos programáticos del IGN, Aguilera delinea un programa de acción que contempló un ambicioso programa editorial y la publicación de un boletín oficial, mismo que apareció por primera vez en 1895 como "Boletín de la Comisión Geológica de México", aunque en el pie del escudo aparecía ya el nombre oficial de Instituto Geológico de México (AGUILERA, 1904, pp. 89-90).

Desde luego, el Instituto Geológico de México no sólo heredó los compromisos de la Comisión Geológica sino que los concluyó y publico. Dichas tareas fueron la elaboración de un bosquejo geológico de México, junto con la carta geológica y minera del país. Esas tareas centrales cristalizaron con la publicación de la primera edición de la Carta Geológica y Minera de México escala 1:3`000,000 (1889) y del Bosquejo Geológico de México (1896).

El siguiente objetivo fue ampliar el grupo de investigadores y por primera vez se contrata en México a dos especialistas en paleontología de prestigio internacional: ellos fueron, el alemán Emil Böse en 1898, y el suizo Carl Burckhardt en 1904, lo mismo que al geólogo estadounidense Paul Waitz para apoyar las labores del nuevo instituto y "descubrir las riquezas geológicas de nuestra Patria, y señalarlas a quienes pudieran explotarlas, beneficiándose y beneficiando a México" (AGUILERA, 1896, p. 125; RUBINOVICH, 2000; GÓMEZ-CABALLERO, 2005, p. 152).

José G. Aguilera fue el heredero y continuador de la labor desarrollada por Antonio del Castillo. Aguilera no sólo le otorgó sustento y prestigio internacional al trabajo científico desarrollado en el Instituto Geológico Nacional, ya que entre otras cosas viajó por el mundo llevando su representación y asistiendo a congresos de su especialidad como los celebrados en Washington, Lieja, San Petersburgo, París, Strasburgo, Viena, Stokolmo; también fungió como el gran articulador de los interese de la comunidad científica nacional dedicada a los estudios mineralógicos, geológicos y paleontológicos, por lo que fue considerado por sus contemporáneos como el regenerador de la geología en México (ORDÓÑEZ, 1938, p. 114; CAPEL, 1992, pp. 168-176). Su biógrafo Alberto Ma. Carreño lo considera el "padre y fundador" de la Geología moderna de México (CARREÑO, 1938, p. 124).

Su obra es basta. Incluye el cultivo de todas las áreas de la geología. En Distribución Geográfica y Geológica de los criaderos minerales de la República estudia la distribución geográfica de la riqueza minera de México; analiza los nichos y las condiciones geoló-

(8) José G. Aguilera, "Estudios de los fenómenos Sísmicos del 3 de mayo de 1887, por J. G. Aguilera”. Anales del Ministerio de Fomento, México, vol. X, p. 7. 
gicas en que se encuentran los criaderos auríferos, auro-argentíferos y argentíferos, los plumbíferos y cupríferos, los de antimonio y de zinc, los de fierro y de manganeso; los criaderos de grafito, de carbón de piedra, de hidrocarburos líquidos, viscosos y sólidos; de ópalo, de amianto, de topacio, de granate; los de azufre, selenio, teluro, y flouro; los de sal, barita astronciana y de caolín; los de cromo, vanadio, estaño; de bismuto y moibdeno; de antimonio, mercurio y cinabrio, etc.

Al cerrar el siglo XIX, en 1898, José G. Aguilera enumeró más de doscientas especies minerales y más de dos mil localidades mineralógicas. En ese momento se habían descubierto una veintena de minerales nuevos en el territorio que eran codiciados por los coleccionistas y por los museos de la especialidad en el mundo. Para entonces la mineralogía como la geología eran disciplinas bien establecidas en el país, tanto desde el punto de vista práctico como científico.

De acuerdo con Morán-Zenteno y Lomnitz (2000), hay tres hechos sobresalientes que indican el dinamismo de las geociencias en México en la primera década del siglo XX, lideradas fuertemente por José G. Aguilera: 1) el inicio de la participación de científicos profesionales en la exploración de hidrocarburos con la localización del pozo Pez № 1 , el cual fue perforado y terminado en abril de 1904 con una profundidad total de $550 \mathrm{~m}$, hecho que marca el inicio de la producción petrolera comercial en México; 2) la fundación de la Sociedad Geológica Mexicana (SGM) en 1904; y 3) la celebración del X Congreso Geológico Internacional en la Ciudad de México en 1906, que tuvo entre otras consecuencias la preparación de 31 libros-guía de excursiones geológicas de diferentes regiones de nuestro país, así como la enriquecedora presencia de investigadores de diversos países (GONZÁLEZ-TORRES, 2004, p. 125; DE CSERNA, 1990, pp. 01-20).

En especial, la Sociedad Geológica Mexicana se formó oficialmente el 6 de diciembre de 1904 y reunió a un compacto grupo de geólogos y mineralogistas mexicanos y extranjeros, con un objetivo común: impulsar los trabajos de investigación de manera colectiva sobre la ciencia de la Tierra y divulgar los frutos de sus resultados entre los funcionarios públicos encargados de elaborar las políticas públicas; entre los actores económicos directamente vinculados con la minería y la agricultura, asimismo, acercar los nuevos conocimientos y saberes a una población ávida de información sobre los recursos naturales de sus comunidades de origen, municipios o regiones ${ }^{(9)}$. Se trata, desde luego, de una sociedad científica especializada que como otras ya existentes en México, dejaba atrás los principios del asociacionismo decimonónico (CAPEL, 1992, pp. 168-176).

El equipo de trabajo que daría sentido a la SGM estuvo precedido por el ingeniero José G. Aguilera, quien fuera su fundador y primer director, y el de mayor ascendencia

(9) En 1904 José G. Aguilera convoca y crea la Sociedad Geológica Mexicana y, con ella, el Boletín de la Sociedad Geológica Mexicana, a tras luz de las siguientes primicias: "México es un país eminentemente minero y agricultor; para estas dos industrias, la base científica es la geología. Hay ya muchos trabajos sobre la constitución geológica del suelo de nuestro país, pero todavía no está organizado el trabajo de los aficionados; una multitud de observaciones útiles se pierden por falta de una publicación que reúna todos los artículos formales, como las noticias y observaciones aisladas y las revistas de la literatura geológica relativa a México. Además, como no hay nada que ligue a los aficionados a la geología entre sí, y que les ponga en contacto, el trabajo personal ha quedado hasta ahora aislado, y muchas veces inadvertido para el mundo científico". Boletín de la Sociedad Geológica Mexicana, tomo 1, julio-diciembre de 1904, México, 1905 , p. 4. 
en la disciplina de la ciencia geológica mexicana de finales del siglo XIX y comienzos del XX (URIBE, 2006). El Ing. Aguilera estuvo al frente de la SGM entre 1904 y 1908, y 1910-1913, y como director del Instituto hasta 1914, en que pasó a ocupar el puesto de Subsecretario de Fomento del gobierno federal ${ }^{(10)}$. En ese tiempo, se publicaron 31 volúmenes del Boletín del Instituto y 8 números del Boletín de la Sociedad Geológica Mexicana (GONZÁLEZ-TORRES, 2004, pp. 123-136) ${ }^{(11)}$.

En los primeros diez años del siglo XX algunos de los profesores relacionados con la enseñanza de la geología en el sistema de escuelas e institutos estatales, es decir, fuera de la ciudad de México, se encontraban vinculados al grupo científico de geólogos del Instituto Geológico Nacional y participarían abiertamente en el desarrollo de las ciencias de la Tierra, otorgando a la disciplina geológica un sustento y una proyección nacional e internacional (URIBE, 2006, pp. 1177-1188).

\section{CONCLUSIÓN}

El sabio y naturalista Andrés Manuel del Río es el artífece de la transición conceptual entre los neutunistas y los plutonistas en México (1821-1849); Antonio del Castillo, discipulo de Del Río, es un punte en la institucionalización de la concepción moderna de la geología cultivada por Charles Leyll; entre la pre-geología y la geología moderna; entre una práctica científica supeditada a la minería a una disciplina cognoscitiva autónoma incorporada como tal a los planes y programas de estudio, y legalmente aceptada en sus implicaciones epistemológicas y políticas en los programas de gobierno. Es, en fin, un inteligente lector de la realidad social y cultural del país; traductor y conciliador de los intereses de la comunidad científica con los intereses del poder político y económico, quien en distintos niveles y a su manera buscaban concretar la ecuación para alcanzar el desarrollo material de México y el fomento científico. En ese escenario, José G. Aguilera, discípulo destacado de Antonio del Castillo, es el gran reformador de los estudios geológicos mexicanos, sólido baluarte de su profesionalización epistemológica y proyección internacional.

José G. Aguilera fue el heredero y continuador de la labor desarrollada por Antonio del Castillo, y este a su vez receptor y también heredero del bagaje científico de Andrés Manuel del Río. De hecho, el trabajo de estos tres hombres representa el sustento teórico y metodológico tanto de la mineralogía como de la geología moderna en México. Sus vidas representan también el largo proceso de institucionalización y profesionalización de las Ciencias de la Tierra, primero a través del Real Seminario de Minería, pasando después por la Escuela Nacional de Ingenieros, para terminar en el Instituto de Geológico Nacional, antecedente directo del actual Instituto de Geología de la Universidad Nacional Autónoma de México.

(10) Su trabajo docente lo realizó a partir de 1909 cuando formó parte del Consejo Superior de Educación Pública; después enseña en la Escuela Nacional Preparatoria, Escuela Nacional de Agricultura, Colegio Militar, etc. La UNAM le otorgó el Honorís Causa el 14 de enero de 1937.

(11) Las publicaciones indicadas constituyen uno de los cimientos fundamentales de la evolución de la geología en México, a través de las cuales se proporcionaron contribuciones diversas en las áreas del conocimiento geológico: descripciones generales sobre la estratigrafía, distritos mineros y localidades fosilíferas. 


\section{BIBLIOGRAFÍA}

AGUILAR Y SANTILLAN, Rafael. Bibliografía Geológica y Minera de la República Mexicana. México: Imprenta Tipográfica de la Secretaría de Fomento, 1898.

AGUILERA,José Guadalupe. Boletín de la Sociedad Geológica Mexicana. México: t. 01, julio-diciembre de 1904, México, 1905.

AGUILERA, José Guadalupe. Bosquejo geológico de México. México: Oficina Tipográfica de la Secretaria de Fomento, 1896.

AGUILERA, José Guadalupe. Estudios de los fenómenos Sísmicos del 03 de mayo de 1887. Anales del Ministerio de Fomento. México: v. X, 1887.

AGUILERA, José Guadalupe. Reseña del desarrollo de la Geología en México. Boletín de la Sociedad Geológica Mexicana. t. 01, n. 01, 1904.

AGUILERA, José Guadalupe; DEL CASTILLO, Antonio. In: AGUILERA, José Guadalupe. Bosquejo Geológico de México. México: Instituto Geológico de México, Oficina Tipográfica de la Secretaría de Fomento, 1896.

ÁRBOL Y BONILLA, José. Programa de la clase de cosmografía con prácticas en el observatorio. Zacatecas: 05 de enero de 1905.

ÁRBOL Y BONILLA, José. Programa de la clase nociones de geología. Zacatecas: 08 de enero de 1905. ÁRBOL Y BONILLA, José. Programa de la clase nociones de meteorología y climatología. Zacatecas: 10 de enero de 1905.

ÁRBOL YBONILLA, José. Programa de la clasenociones de mineralogía. Zacatecas: 17 de enero de 1905. AZUELA BERNAL, Luz Fernanda y GUEVARA FEFER, Rafael. La ciencia en México en el siglo XIX: una aproximación historiográfica. Asclepio, Revista de Historia de la Medicina y de la ciencia, Madrid, v. 50, n. 02, 1998.

AZUELA BERNAL, Luz Fernanda. La institucionalización de la meteorología en México a finales del siglo XIX. In: La cultura científico-tecnológica en México: Nuevos materiales multidisciplinarios. México: UNAM, 1995.

AZUELA BERNAL, Luz Fernanda. Tres sociedades científicas en el Porfiriato. Las disciplinas, las instituciones y las relaciones entre la ciencia y el poder. México: SMHCyT-UTN-UNAM, 1996.

AZUELA BERNAL, Luz Fernanda. De las minas al laboratorio: la demarcación de la Geología en la Escuela Nacional de Ingenieros (1795-1895). México: Instituto de Geografía, Facultad de Ingenieros, UNAM, 2005.

BÁRCENA, Mariano. Datos para el estudio de las rocas mesozoicas de México y sus fósiles característicos. Sociedad Mexicana Geografía Estadística. v. 03, n. 02, 1875.

BÁRCENA, Mariano. Descripción de un crustáceo fósil del género Spheroma (S. burkartii) y reseña geológica del Valle de Ameca, Jalisco. La Naturaleza. Periódico Científico de la Sociedad Mexicana de Historia Natural, v. 03, 1875.

BARGALLÓ, Modesto. Andrés Manuel del Río y el Bicentenario de su nacimiento (1764) (Su labor Geológica, Mineralógica y Minerometalúrgica). Revista de la Sociedad Mexicana de Historia Natural. v. 25, 1964.

BARGALLÓ, Modesto. Conveniencia de sustituir el nombre de Vanadium por el de Erythronium. In: PRIETO, C.; SANDOVAL VALLARTA, M.; BARGALLÓ, M. y ARNÁIZ Y FREG, A. Andrés Manuel del Río y su obra científica. Segundo centenario de su natalicio (1764-1849). México: Cia. Fundidora de Fierro y Acero de Monterrey, 1966. 
BAZANT, Mílada. La enseñanza y la práctica de la ingeniería durante el Porfiriato. Historia Mexicana. n. 131, El Colegio de México, 1984.

BELTRAN, Enrique. El Primer Centenario de la Sociedad Mexicana de Historia Natural (1868-1968). Revista de la Sociedad Mexicana de Historia Natural. v. 29, 1968.

CAPEL, Horacio. El asociacionismo científico en Iberoamérica. La necesidad de un enfoque globalizador. Interciencia. v. 17, n. 03, Caracas, 1992.

CARPY NAVARRO, Patricia Justina Guadalupe. La Sociedad Mexicana de Historia Natural y su influencia en el siglo XIX. México: tesis para obtener el titulo de Licenciado en Historia. Facultad de Filosofía y Letras, UNAM, 1986.

CARREÑO, Alberto Ma. Un insigne Geólogo Mexicano. Discurso pronunciado en la velada efectuada el 04 de febrero de 1937, en el seno de la Sociedad Geológica Mexicana, por el Sr. Prof. Alberto Ma. Carreño, Delegado de las Sociedades Científicas de México. Boletín de la Sociedad Geológica Mexicana. t. 10, n. 03-04, 1938.

CARREÑO, Ana Luisa y MONTELLANO-BALLESTEROS, Marisol. La Paleontología mexicana; pasado, presente y futuro. Boletín de la Sociedad Geológica Mexicana. t. 57, n. 02, 2005.

CARRERA STAMPA, Manuel Antonio del Castillo, 1820-1895. Gacetas Históricas. Congreso Geológico Internacional, XX Sesión, México: Editorial Jus, 1956.

CASTAÑEDA CRISOLES, Edgar. Practicas y enseñanza de la ingeniería civil en el Estado de México, 1870-1900. Ponencia en el III Coloquio de Historia de la Ciencia y la Tecnología Regional. Guanajuato: septiembre de 1995.

CASTILLO MARTOS, Manuel. Creadores de la ciencia moderna en Españay América. Ulloa, los Delhuyar y del Río descubre el platino, el wolframioy el vanadio. España: Muñoz Moya Editores Extremeños, 2005.

CUEVAS CARDONA, María del Consuelo. Un científico mexicano y su sociedad en el siglo XIX, Manuel María Villada, su obra y los grupos de los que formó parte. México: Universidad Autónoma del Estado de Hidalgo, Sociedad Mexicana de Historia de la Ciencia y la Tecnología, 2002.

DE CSERNA, Zoltan. La evolución de la Geología en México (1500-1929). Revista del Instituto de Geología. v. 09, n. 01, 1990.

DEL CASTILLO, Antonio. Adelantos de la paleontología y geología del Valle de México. El Minero Mexicano, 1879.

DEL CASTILLO, Antonio. Carta Geológica y Minera de México. Escala 1:3`000,000, 1889.

DEL CASTILLO, Antonio. Catálogo de las especies minerales y sus variedades que se encuentran en México. Cuadro de la mineralogía mexicana, conteniendo las especies minerales dispuestas por orden de su composición química y cristalización, con arreglo al sistema del Profesor Dana. Boletín de la Sociedad Mexicana de Geografía y Estadística. n. 01, 1864.

DEL CASTILLO, Antonio. Clasificación y datos sobre los mamíferos fósiles encontrados en el Valle de México. Deutsche Geologische Gessellschaft, Zeitschrift, n. 21, 1869.

DEL RÍO, Andrés. Sobre la formación de las montañas de algunos reales de minas. Gaceta de México. México: Suplemento, 1803.

DEL RÍO, Andrés. Manual de Geología extractada de la Lethaea geognostica de Bronn con animales y vegetales perdidos o que ya no existen, mas característicos de cada roca, y con algunas aplicaciones a los criaderos de esta República para uso del Colegio Nacional de Minería. México: Imp. I. Cumplido, 1841.

DEL RÍO, Andrés. Mineralogía. Carta dirigida al señor Abate Hauy, canónogo honorario de la Santa Iglesia de París, de la Legión de Honor y del Instituto, profesor de minerañlogía, etc., etc., por D. Andrés del Río, de la Sociedad Económica de Lipsia, y de otras extranjeras, corresponsal de la Academia Médica Matritense, etc. El Seminario Político y Literario, v. 02, n. 83, 1820-1821. 
DEL RÍO, Andrés. Suplemento de Adiciones y correcciones de mi Mineralogía, impresa en Filadelfia en 1832; esto es, diez y seis años hace, en cuyo tiempo se han hecho en Europa y en los Estados-Unidos varios descubrimientos que les importa saber a los alumnos de Minería, por el Ciudadano Andrés del Río. Profesor jubilado por el Supremo Gobierno, y corresponsal del Instituto Nacional de Francia, y de otras Academias y Sociedades Científicas. México: Tipografía de R. Rafael, 1848.

DEL RÍO, Andrés; HERRERA, M.: T. R. del Moral. Informe sobre la porcelana de Puebla. Siglo XIX, v. 02, n. $532,1843$.

Diario Oficial, t. XIX, n. 152, México, 25 de diciembre de 1888.

DÍAZ Y OVANDO, Clementina. Los veneros de la ciencia mexicana. Crónica del Real Seminario de Minería (1792-1892). México: Facultad de Ingeniería, Universidad Nacional Autónoma de México, tomos I, II, III, 1998.

DUBLAN, Manuel y LOZANO, José María. Legislación mexicana o colección completa de las disposiciones legislativas expedidas desde la independencia de la república. México: Imprenta del Comercio, tomo 06, 1876-1911.

FLORES CLAIR, Eduardo. Minería, educación y sociedad. El Colegio de Minería, 1774-1821. México: Colección Científica n. 419, Instituto Nacional de Antropología e Historia. 2000.

FOUCAULT, Michel. Las palabras y las cosas. Una arqueología de las ciencias humanas. Buenos Aires: Siglo Veintiuno editores, 1966.

FOUCAULT, Michel. La arqueología del saber. México: Siglo Veintiuno editores, 1979.

FOUCAULT, Michel. El orden del discurso. Buenos Aires: Tusquets Editores, 1992.

GALINDO Y VILLA, Jesús. D. Antonio Á Castillo. La Naturaleza. Periódico Científico de la Sociedad Mexicana de Historia Natural, Segunda serie, t. 03, Años de 1897 a 1903. México: Imprenta de Ignacio Escalante, 1903.

GALVEZ CAÑERO, Alzola, A. Apuntes biográficos de Fausto de Elhuyar y de Zubice. Boletín del Instituto Geológico y Minero. t. 53, n. 08, 1933.

GÍO-ARGÁEZ, Raúl y RODRÍGO ARÉVALO, Yunuen. Panorama General de la Paleontología Mexicana. Ciencia Ergo Sum. Revista científica multidisciplinaria, v. 10, n. 01, 2003.

GÓMEZ CABALLERO, J. Arturo. Historia e índice comentado del Boletín del Instituto de Geología de la UNAM. Boletín de la Sociedad Geológica Mexicana, t. 57, n. 02, 2005.

GONZÁLEZ-TORRES, Enrique. Bosquejo sobre la evolución de la Geología en México (1904-2004). Boletín de la Sociedad Geológica Mexicana, t. 57, n. 02, 2004.

LYELL, Charles. Principles of Geology. London. 3 v. 1830.

Memoria presentada a S. M. El Emperador por el Ministro de Fomento Luís Robles Pezuela de los trabajos ejecutados en su ramo en el año de 1865. México: Imprenta de J. M. Andrade y F. Escalante, 1866.

MORÁN-ZENTENO, D. y LOMNITZ. C. Las ciencias de la tierra en México. In: MENCHACA, A. (coord.). Las ciencias exactas en México. México: Consejo Nacional para la Cultura y las Artes, Fondo de Cultura Económica, Fondo de Estudios e Investigaciones Ricardo J. Zevada, 2000.

MORELOS RODRÍGUEZ, Lucero. La Geología Mexicana en el Siglo XIX. Una revisión histórica de la obra de Antonio del Castillo, Santiago Ramírezy Mariano Bárcena. México, Plaza y Valdés, 2012.

MORELOS RODRÍGUEZ, Lucero. Antonio del Castillo (1820-1895) y su contribución al conocimiento de las ciencias de la tierra en el siglo XIX. México: UMSNH, 2013.

ORDÓÑEZ, Ezequiel; AGUILERA, José G. Discurso pronunciado en la velada efectuada la noche del 4 de febrero de 1937, en el seno de la Sociedad Geológica Mexicana, por el Sr. Ing....”. Boletín de la Sociedad Geológica Mexicana, t. 10, n. 03-04, 1938. 
PALACIOS, Leopoldo. Importancia de la Ingeniería en México. México: Tipografía Vda. de F. Díaz de León, 1911.

PELAYO, Francisco y REBOK, Sandra. Fausto de Elhuyar y la Societat der Bergbaukunde. Un proyecto científico de red europea para la difusión de las prácticas minero-metalúrgicas. Cronos. v. 05-06, 2002-2003.

PESET, José Luis. Ciencia y Libertad. El papel del científico ante la independencia americana. Madrid: CSIC, 1987.

RAMÍREZ, Santiago. Datos para la Historia del Colegio de Minería. México: Imprenta del Gobierno Federal en el ExArzobispado, 1890.

ROBLES, V. A. El Ilustre Maestro Andrés Manuel del Río. México: s/e, 1937.

ROBLES BERUMEN, Ciro Robles. Difusión e institucionalización de la ciencia moderna en Zacatecas (1732-1850). Protocolo de tesis doctoral, Programa de Doctorado en Historia, Universidad Autónoma de Zacatecas, 2001.

RUBINOVICH KOGAN, Raúl. Andrés Manuel del Río y sus Elementos de Orictognosia de 1895-1805. In: Andrés Manuel del Río, Elementos de Orictognosia, 1795-1805. Edición y estudio introductoria: Raúl Rubinovich Kogan. México: Universidad Nacional Autónoma de México, 1992.

RUBINOVICH KOGAN, Raúl. Ezequiel Ordoñez. Vida y obra. México: El Colegio Nacional, 2000.

SALDAÑA, Juan José. Ciencia y felicidad pública en la Ilustración americana. In: SALDAÑA, JUAN JOSÉ (Coordinador). Historia social de las ciencias en América Latina. México: Grupo Editorial Miguel Ángel Porrua, Universidad Nacional Autónoma de México, 1996.

URIBE SALAS, José Alfredo; CORTÉS ZAVALA, María Teresa. Andrés del Río, Antonio del Castillo y José G. Aguilera en el desarrollo de la ciencia mexicana del siglo XIX. Revista de Indias. v. 66. n. $237,2006$.

URIBE SALAS, José Alfredo. Labor de Andrés del Río en México: profesor en el Real Seminario de Minería e innovador tecnológico en minas y ferrerías. Asclepio. Revista de Historia de la Medicina y de la Ciencia, Departamento de la Ciencia. España: CSIC, 2006.

URIBESALAS, José Alfredo. Práctica científica y descubrimiento del Vanadio en la biografía de Andrés del Río. URIBE SALAS, JOSÉ ALFREDO (Coordinador). Estudios de Historia Regional Continental. México: UMSNH, 2008.

URIBE SALAS, José Alfredo. Andrés Manuel del Río: formación científica y desempeño académico en el Real Seminario de Minería de México. Ciencia Nicolaita. Revista de la CIC, México: UMSNH, n. 46, abril de 2007.

URIBE SALAS, José Alfredo. La Escuela Nacional de Ingenieros y la formación de profesionistas para el desarrollo de la economía de México, siglo XIX. In: Historia de la Educación en México. México: El Colegio de San Luís, formato CD, 2001.

URIBE SALAS, José Alfredo. La Sociedad Geológica Mexicana, 1904-1912. Un eslabón en la profesionalización de las Ciencias de la Tierra en México. In: PÉREZ BUSTAMANTE; Juan Antonio; MARTÍN FERNÁNDEZ; Cándido (Editores). Actas del IX Congreso de la Sociedad Española de Historia de las Ciencias y de las Técnicas. Cádiz: Sociedad Española de Historia de las Ciencias y de las Técnicas, 2006.

URIBE SALAS, José Alfredo. De la aclimatación de la Mineralogía al desarrollo de la Geología o la promoción de conocimientos para el desarrollo de México, Siglo XIX. In: CALVO, Luis; GUIRÓN SIERRA, Alvaro; PUIG SAMPER, Miguel Ángel (Editores). Naturaleza y Laboratorio. Barcelona: CSIC, 2013.

VILLASEÑOR, Lorenzo. Compendio de mineralogía, adatado como texto de la clase de químicay nociones de mineralogía en el Instituto de Ciencias del Estado de Zacatecas. Zacatecas: Imprenta Literaria, 1910. 